\title{
O SIGNIFICADO DA ASSISTÊNCIA DE ENFERMAGEM SEGUNDO ABORDAGEM DE ALFRED SCHÜTZ
}

\author{
THE SIGNIFICANCE OF THE NURSING ASSISTANCE SECOND \\ ALFRED SCHÜTZ
}

\author{
EL SIGNIFICADO DE LA ASISTENCIA DE ENFERMERÍA SEGÚN \\ ENFOQUE DE ALFRED SCHÜTZ
}

\author{
Miriam Marinho Chrizostimo* \\ Ann Mary Machado Tinoco Feitosa Rosas ${ }^{* *}$ \\ Luizélia Alves ${ }^{* * *}$ \\ Miriam Gentil Bartoly ${ }^{* * * *}$ \\ Carlos Magno Carvalho da Silva ${ }^{* * * *}$ \\ Enilda Moreira Carvalho Alves ${ }^{* * * * *}$
}

\begin{abstract}
RESUMO
Trata-se de um artigo de revisão bibliográfica que busca uma articulação entre a assistência de enfermagem e a fenomenologia de Schütz. Objetiva-se revisar o significado da assistência de enfermagem segundo a abordagem fenomenológica de Schütz, que se fundamenta na ação do enfermeiro, na motivação e razões. O referencial teórico está apoiado na fenomenologia sociológica de Schütz. O estudo apontou os motivos-para e os motivosporque para a compreensão do significado dessa assistência; o primeiro destaca a recuperação, promoção da saúde, conforto, bem estar e segurança do cliente; o segundo ressalta a importância do ambiente e condições adequadas para o desenvolvimento da assistência. São necessários modelos de gestão participativa e estratégias inovadoras para a transformação da prática e o desenvolvimento do cuidar holístico e humanizado com qualidade, pautados no paradigma sanitário.
\end{abstract}

Palavras chave: Atenção de Enfermagem, enfermeira, filosofia em enfermagem.

\begin{abstract}
This article is a review of the literature looking for a relationship between nursing and Schütz phenomenology Model. Its aim is to examine the meaning of nursing care according to Schütz's phenomenological approach, based on the actions of nurses, their motivations and reasons. The theoretical framework is based on the sociological phenomenological of Schütz. The study found that the "reasons for" and "reasons why" to understand the

\footnotetext{
* Enfermeira, Professora Mestre do Departamento de Enfermagem de Fundamentos de Enfermagem e Administração da Escola de Enfermagem Aurora de Afonso Costa da Universidade Federal Fluminense. miriammarinho@hotmail.com / profmiriammarinho@yahoo.com.br

** Enfermeira. Docente Doutora do Departamento de Metodologia da Enfermagem da Escola de Enfermagem Anna Nery da Universidade Federal do Rio de Janeiro.

${ }^{* * *}$ Enfermeira Supervisora da Divisão de Supervisão da Rede Contratada e Conveniada do Departamento de Supervisão Técnica-metodológica da Fundação Municipal de saúde de Niterói.

${ }_{* * * *}$ Enfermeira Supervisora da Divisão de Supervisão da Rede Própria do Departamento de Supervisão Técnica-metodológica da Fundação Municipal de saúde de Niterói.

${ }_{* * * * *}$ Enfermeiro graduado pela Universidade Federal Fluminense. Discente do Mestrado Acadêmico em Ciências do Cuidado em Saúde (MACCS). Escola de Enfermagem Aurora de Afonso Costa (EEAAC/UFF) mcarvalho27@yahoo.com.br

${ }_{* * * * * *}$ Enfermeira, Professora Mestre, do Departamento de Enfermagem de Fundamentos de Enfermagem e Administração da Escola de Enfermagem Aurora de Afonso Costa da Universidade Federal Fluminense. enildacarvalhoalves@yahoo.com.br
} 
meaning of care; the first one emphasizes the recovery, health promotion, comfort, welfare and safety of the client; the second, emphasizes the importance of the environment and conditions for assistance development. Participatory management models and innovative strategies for transforming the practice and development of integral and humanized care are neccesary, guided by the health paradigm.

Key words: Nursing care, nursing, philosophy in nursing.

\section{RESUMEN}

Este es un artículo de revisión de la literatura que busca una relación entre la atención de enfermería y la fenomenología de Schütz. Su objetivo es examinar el significado de la atención de enfermería según la aproximación fenomenológica de Schütz, que se basa en la acción de las enfermeras, sus motivaciones y razones. El marco teórico se apoya en la fenomenología de Schütz. El estudio encontró los "motivos-para" y "motivospor qué" para comprender el significado de la asistencia; el primero destaca la recuperación, promoción de la salud, la comodidad, el bienestar y seguridad del cliente; el segundo hace hincapié en la importancia del medio ambiente y las condiciones al desarrollo de la asistencia. Se necesitan modelos de gestión participativa y estrategias innovadoras para la transformación de la práctica y el desarrollo del cuidado integral y humanizado, guiados por el paradigma en la salud.

Palabras clave: Atención de enfermería, enfermera, filosofía en enfermería.

Fecha recepción: 01/10/07 Fecha aceptación: 06/11/09

\section{INTRODUÇÃO}

Este estudo foi construído a partir de revisão bibliográfica, na busca da articulação entre a assistência de enfermagem e a fenomenologia sociológica proposta por Schütz (1).

A assistência de enfermagem cogita a sustentação das práticas de saúde tão necessárias no cotidiano do cliente, tendo em vista que o cuidar é uma das ferramentas do processo de trabalho que o enfermeiro dispõe para aplicação do conhecimento técnico-científico, imprescindível à assistência ao usuário e otimização das suas ações.

Por tanto, a assistência contribui efetivamente para que o exercício profissional do enfermeiro seja visto pela sociedade como arte do cuidar, deslocando-a para ciência que aponta para uma metodologia própria por meio do saber técnico-científico.

Desse modo, os profissionais de saúde desenvolvem suas práticas a partir de competências adquiridas por meio de um processo de formação que tem por base o acúmulo e desenvolvimento de conhecimentos e de tecnologias (2). Observa-se, então, que a ampliação do saber humano é um processo histórico, varia de acordo com o momento, espaço e a prática que orienta o saber-fazer dos profissionais, principalmente o enfermeiro, o que faz com que a assistência de enfermagem valorize a singularidade do ser humano, o cliente.

Dessa forma, a proposta se justifica pela compreensão do significado da assistência de enfermagem por meio da abordagem de Schütz, pois essa abordagem possibilita compreender a vida social, o conjunto de ações sociais o qual as relações mútuas são estabelecidas de maneira conscienciosa (1).

A partir de tal constatação, deu-se início à busca do material bibliográfico com relação à assistência de enfermagem e a abordagem de Schütz (1), quando emergiram os seguintes questionamentos: $\mathrm{O}$ que se tem em vista quando é prestada assistência de enfermagem? Qual é o significado dessa atividade assistencial para o enfermeiro?

Nesse contexto, foi possível traçar o objetivo desta pesquisa: compreender o significado da assistência de enfermagem mediante 
a abordagem fenomenológica de Schütz (1). Espera-se que este estudo propicie a ampliação da discussão crítica-reflexiva sobre o tema e, assim, auxiliar na construção de modelos de gestão participativa. O entendimento do significado colabora para o alcance da eficiência e eficácia da ação do enfermeiro no processo do cuidar.

\section{MATERIAL E MÉTODO}

O estudo trata de um estudo bibliográfico que por meio da revisão analítica se discute o significado da assistência de enfermagem segundo a abordagem de Schütz(1).

Para realizar esta pesquisa buscou-se a produção científica existente sobre a temática, e para tal foi necessário percorrer os passos especificados a seguir. Primeiramente, a procura da produção científica se deu na Biblioteca Virtual de Saúde(3), considerando as palavras-chave, assistência de enfermagem e qualidade, obtendo-se três trabalhos(4-6).

Em segundo momento, nas Ciências da Saúde em Geral, a partir das palavras-chave abordagem fenomenológica e Schütz(1) foram identificadas dezesseis produções, as quais foram reconhecidas na base de dados Literatura Latino-Americana e do Caribe em Ciências da Saúde (LILACS). Ressalta-se que das dezesseis obras foram utilizadas quatro produções $(1,8,9,10)$ por focalizarem o tema e o paradigma flexneriano ou paradigma sanitário.

Em seguida, a leitura da literatura apoiouse nos autores citados (1-23) neste estudo, cuja análise voltou-se para o assunto, emergindo dois subtemas - assistência de enfermagem e o campo de pensamento de Alfred Schütz (1). Os materiais selecionados para a realização dessa pesquisa foram os artigos, sites, livros, dissertação e tese, o que possibilitou a análise dos conteúdos, de acordo com o objetivo traçado.

\section{A Assistência de Enfermagem}

Na visão fenomenológica, descreve-se a ação do enfermeiro na assistência de enfermagem como um processo fundamentado na motivação, razões e objetivos, guiados por antecipações, na forma de planejamento e projeções desse profissional.

$\mathrm{Na}$ assistência de enfermagem, a ação do enfermeiro neste mundo pode ser vista como um ato de cuidar humanizado, pois quem pratica o cuidado zela, preocupa-se, observa, analisa e cria (10).

O enfermeiro, em sua assistência, deve considerar a consciência pessoal e o pensamento como sensíveis e mutáveis, o que se compara a uma corrente de pensamento, corrente de experiência ou cogitações, corrente de vida pessoal consciente(1).

Nesta conjuntura, percebe-se que a experiência individual do enfermeiro está relacionada ao vivido desse profissional, assim, à medida que ele vive, centra-se nos objetos dessa experiência e perde de vista os atos da experiência subjetiva em si. Para que esses atos sejam revelados, deve-se modificar a atitude ingênua com a qual ele se dirige aos objetos e para o qual volta sua própria experiência, na busca do caminho específico de reflexão(1).

No entanto, para revelar a visão que se chega à intencionalidade da consciência, por meio da ponderação, necessita-se apontar o caráter básico das cogitações, que é o fato de ser consciência de algo. Desta maneira, a ação no mundo da vida está voltada para o desenvolvimento de uma teoria subjetiva, em que ela é vista como um processo de motivação(1).

Desse modo, observa-se que o enfermeiro precisa utilizar a escuta e o diálogo como estratégias do cuidar para adequar sua ação às necessidades do cliente, compreendido como um ser singular. No que se refere à ação junto à equipe de enfermagem, as razões e os objetivos do enfermeiro permitem a educação permanente em saúde, que, atualmente, é consi- 
derada uma tática importante na orientação e capacitação dos profissionais dessa área (11). Por isso afirma-se que a enfermagem moderna envolve o enfermeiro em diferentes tipos de atividades, conceitos relacionados às ciências da saúde e exige competência ética e técnico-científica.

$\mathrm{O}$ enfermeiro necessita compreender que a enfermagem é a arte e a ciência do cuidar, imprescindível à preservação da vida das pessoas (12). Acrescenta-se que essa profissão, a enfermagem, é única à medida que se dedica humana e holisticamente ao atendimento das necessidades humanas básicas de pacientes e familiares, frente aos problemas de saúde reais e potenciais, com autonomia, considerando suas competências(13).

Contudo, nem sempre os fatos ocorreram dessa forma, considerando que, na enfermagem das sociedades antigas, cabia, geralmente às enfermeiras, um papel de subserviência, segundo o qual era proporcionada assistência aos doentes, realizada sob supervisão direta de um médico, portanto, sem autonomia, também cabia a elas zelar pela higiene das casas dos pacientes (13).

Atualmente, para o enfermeiro é primordial exercer a assistência que lhe cabe com qualidade. A qualidade aqui é entendida como possibilidade de alcançar o mais alto padrão de eficiência e eficácia da assistência, isto é, resolutiva e segura, atendendo, de forma singular, a cada cliente.

O enfermeiro precisa entender, de forma clara, sua motivação pessoal e profissional. A pessoa carece ser vista de maneira singular, com suas particularidades, assim como o profissional deve ser percebido em seu vivido acadêmico, em seu contexto histórico-social.

Sendo assim, no que se refere à formação do enfermeiro, um profissional do cuidar, o professor deve atentar para as implicações psicoafetivas, histórico-existenciais e profissionais (14) do graduando, pois, este estudante irá prestar assistência ao cliente de acordo com sua escolha, uma vez que as formas de cuidar dependem daqueles que têm competência e habilidades para implementa-las. Vários estudiosos consideram que a essência da enfermagem é o cuidado, um cuidado implementado pelo enfermeiro ao cliente em todas as dimensões, em especial de sua consciência: o eu, a pessoa, o cidadão e o sujeito $(15,18)$.

Dessa forma, o enfermeiro estará vinculado, efetivamente, à ação do cuidar na instituição e, para que isso possa ser facilitado, ele deve participar da definição do paradigma a ser adotado, especificando o desenho assistencial, missão, visão, princípio, objetivo e meta, o que favorecerá para que se instale o dinamismo institucional que possibilita que esse profissional possa se apropriar e utilizar tais elementos como ferramentas para a gestão dos cuidados aos clientes, do pessoal, dos recursos materiais e ambientais necessários à avaliação da assistência de enfermagem na instituição.

No entanto, o típico da ação da equipe de enfermagem ainda se revela uma ação com olhar biologicista, voltada para orientar / ensinar (7), o que dificulta a implementação da ação no paradigma sanitário.

O profissional de enfermagem tem acesso à pessoa como um todo, em todas as dimensões, nas suas interações com o paciente, família e comunidade. O cuidado de enfermagem envolve a compreensão dos conhecimentos e experiências psicossociais, culturais e econômicas do paciente, de forma a propiciar a manutenção, promoção e adaptação às mudanças geradas pela patologia (19).

Nesse contexto, enfermeiros e demais funcionários do serviço de enfermagem, ao se basearem na realidade que vivenciam, poderão refletir sobre sua problemática para, então, definirem os fundamentos que norteiam suas ações (20). Para tal, faz-se indispensável a implementação de um método de avaliação ou auto-avaliação contínua e construtiva da assistência realizada pelos enfermeiros, quando existe o propósito de aperfeiçoar e qualificar a assistência desses profissionais e otimizar o cuidado aos pacientes. Para isso, o 
enfermeiro precisa compreender o significado da assistência que executa.

\section{O campo de pensamento de Schütz}

Alfred Schütz nasceu em Viena, em 1899, e morreu em Nova York em 1959. Interessouse profundamente pelo trabalho de Weber e Husserl. Como sociólogo, trouxe várias contribuições para o tratamento dos fenômenos de tipificações na esfera cotidiana $(1,21$, 22).

Schütz, profundo conhecedor da filosofia, possibilitou confrontar esta ciência com a visão sociológica, e não com a sociologia como um todo. Marcou um ponto de partida radical, a sociologia da ação e compreensão de Weber. Procurou um método para compreender a vida social, o conjunto de ações sociais no qual as relações mútuas são estabelecidas de maneira conscienciosa $(1,9)$.

O campo de ação do pensamento de Schütz está pautado no conteúdo das idéias, concepções e pode ser subdividido em tópicos, tais como: fundamentos fenomenológicos para o tipo de sociologia que este visava estruturar; estrutura e funcionamento da consciência humana e suas ramificações sociais; estrutura e funcionamento do mundo social como um conjunto de construções mentais e suas duplas raízes na experiência individual e nos padrões preestabelecidos de relacionamentos sociais; características de diferentes domínios da experiência humana; fundamentos teórico-conceituais e metodológicos de uma sociologia de orientação fenomenológica (1).

Assim, ressalta-se que Schütz analisou os fatores fundamentais determinantes do comportamento do indivíduo no mundo da vida; a ação neste mundo da vida é vista como um processo fundamentado em funções de motivação, razões e objetivos, guiados por antecipações na forma de planejamento e projeções (1).

Assim sendo, no quadro da sociologia fenomenológica de Schütz, o esclarecimento sobre $\mathrm{o}$ ato pelo qual a vontade se determina a algo -a escolha, liberdade e determinismo no contexto da ação humana- está incluído em outras subdivisões, tais como: fundamentos da fenomenologia; cenário cognitivo do mundo da vida; mundo das relações sociais; reinos da experiência e a província da sociologia (1).

A abordagem fenomenológica da sociologia compreensiva de Schütz (1) enquanto estratégia teórico-metodológica permite captar o típico da ação a partir do sujeito que vivencia as experiências no mundo da vida (8).

Considerando o exposto, apresenta-se, a seguir, o referencial teórico do estudo, centrado na assistência de enfermagem como um processo fundamentado na ação do enfermeiro e em suas motivação, razões e objetivos profissionais, entendendo que o cuidar desenvolvido pelo Enfermeiro precisa ser percebido como uma ação social (9).

\section{Os motivos-para e os motivos-porque da ação do enfermeiro}

A fenomenologia sociológica, fundamentada no vivenciar a experiência, valoriza a vivência que é única, pois, só o sujeito da ação pode dizer o que pretende sentir na relação com a mesma. Além disso, toda ação intencional tem significado. Ao investigar os impulsos subjetivos por trás da ação humana, é encontrada a teoria da motivação os homens agem em função de motivações (1).

Assim, a partir dos questionamentos deste estudo foram projetados os motivos-para e os motivos-porque da ação do enfermeiro, dando origem a dois entendimentos sobre significado da assistência de enfermagem, descritos a seguir.

O primeiro entendimento projeta os motivos-para. Permite orientar para que o cuidado alcance o potencial do cliente no que se refere ao físico-social, ultrapassando o limite do que é exteriorizado. O segundo deve possibilitar assistência de qualidade para atingir 
a singularidade do cliente.

No primeiro, a ação do enfermeiro procura o que não está contido na aparência. Compreende o significado da essência do cuidar. Leva-se em consideração ainda a recuperação, a promoção da saúde, conforto, bem-estar, consolo e aproximação com o outro.

No segundo entendimento, a ação do cuidar do profissional enfermeiro volta-se para a segurança, fundamentada no conhecimento ético, técnico e teórico-científico que embasam a profissão e a maneira de cuidar, valorizando a singularidade do cliente.

No que se refere aos motivos-porque, esses se manifestam de duas maneiras. Primeiramente o processo de trabalho do enfermeiro é possuidor de carga histórica, cultural, política e ideológica. E, em outro momento, o processo de trabalho com dependência do ambiente e condições de trabalho.

Com essa reflexão, nota-se que, apesar do paradigma sanitário estar presente no cotidiano dos enfermeiros, ainda permanece, na prática, o reflexo do biologicismo. Porém, entende-se que o conceito de saúde, em sua contextualização, encontra-se com a relação social; a ausência de doença não o restringe.

Percebe-se que a visão de mundo do homem modifica seu comportamento e cria nova expectativa, de acordo com o seu vivido e sua vivência na formação acadêmica e no seu processo de vida pessoal. Entretanto, para que possa ocorrer a realização da assistência de qualidade e outras atividades, é preciso que o enfermeiro tenha competência, ética, conhecimento técnico-científico e condições para o exercício profissional.

Por conseguinte, a vivência e o vivido do enfermeiro não se restringem a algo isolado. O conjunto, formado pelo meio onde se vive e a forma do viver, reflete diretamente em suas ações que revelam o sujeito ativo, portador de concepções, costumes e comportamentos e de determinadas formas de pensar e atuar sobre a realidade $(21,22)$.

Assim, a qualidade e a eficácia da atenção dispensada aos usuários dos hospitais públicos no Brasil deve ser realizada por profissionais capacitados para haver a conceituação que valorize a vida humana e a cidadania dentre outros aspectos importantes para o tratamento humanizado (23).

\section{RESULTADOS E DISCUSSÃO}

Ao discutir este estudo afirma-se que a análise crítico-reflexiva da literatura revisada foi realizada, tendo em vista que o significado da assistência de enfermagem emergiu quando os questionamentos iniciais foram respondidos.

Desse modo, quando as questões vieram a tona, percebeu-se que os motivos-para e os motivos-porque estão envolvidos nas questões referentes ao que o enfermeiro tem em vista quando presta assistência a clientela e no significado dessa atividade assistencial para este enfermeiro.

Sendo assim, os motivos-para compreende a essência do cuidar e implica a recuperação, a promoção da saúde, conforto, bem-estar, consolo e aproximação do outro, refere-se à segurança, fundamentada no conhecimento ético e técnico-científico da profissão.

O segundo significado, estão nos motivos-porque, pois se referem ao processo de trabalho vivido pelo enfermeiro, que tem sentido histórico, cultural, político e ideológico. Como também, depende do ambiente e condições adequadas e seguras de trabalho.

Por isso, constata-se que a compreensão do significado da assistência de enfermagem traz a concepção da saúde, a qualidade da assistência e a necessidade de atendimento da Política Nacional de Humanização.

Assim, é preciso qualificar as ações da assistência à saúde; ser eficiente na atenção dispensada aos usuários dos hospitais públicos no Brasil; capacitar os profissionais dos hospitais para um novo conceito de assistência à saúde, a qual valorize a vida humana e a cida- 
dania; conceber e implantar novas iniciativas de humanização dos hospitais que venham beneficiar os usuários e os profissionais de saúde; fortalecer e articular todas as iniciativas de humanização já existentes na rede hospitalar pública; estimular a realização de parcerias e intercâmbios de conhecimentos e experiências nesta área; desenvolver um conjunto de indicadores de resultados e sistema de incentivos ao tratamento humanizado.

Dessa forma, o objeto ideal, a partir de uma realidade criada na consciência do enfermeiro, não é suficiente, ao contrário, é preciso que os vários atos da consciência sejam conhecidos em suas essências. De tal modo, essa realidade não é única, mas comum à concepção dos demais, considerando que a vivência, na singularidade de cada ser, tornase um caminho percorrido por enfermeiros com experiências diversas, em tempos diferentes de atuação.

Vale ressaltar que toda vivência intencional é um fenômeno. Como fenômeno designa, em sua concepção, um objeto aparente e, como tal, isto implica que toda vivência intencional não só se refere a objetos, mas que ela mesma é objeto de certas vivências intencionais.

A partir deste ponto, afirma-se que tais considerações são pertinentes, tendo em vista que a compreensão do significado representa o desvelar da intencionalidade e a essência da consciência do enfermeiro.

\section{CONCLUSÕES}

O entendimento do aspecto de concepção da saúde e do sentido de qualidade da assistência possibilitam o movimento de criação de estratégias inovadoras para a transformação da prática profissional do enfermeiro, superando o paradigma flexneriano e fortalecendo o paradigma sanitário para o desenvolvimento do cuidar na conjuntura da atenção holística e humanizada com qualidade.

\section{REFERÊNCIAS}

1. Schütz A.Textos escolhidos de Alfred Schütz: fenomenologia e relações sociais. Rio de Janeiro: ZAHAR Editores; 1979, 139.

2. Ministério da Saúde (Br). Secretaria de Gestão do Trabalho e da Educação na Saúde. Curso de Facilitadores de Educação Permanente em Saúde: unidade de aprendizagem - análise do contexto da gestão e das práticas de saúde. Rio de Janeiro: Brasil. Ministério da Saúde/FIOCRUZ; 2005.

3. Biblioteca Virtual de Saúde. Encontrada em http://.bvsms.saude.gov.br Acesso em 18 de setembro de 2007.

4. Matos E, Pires D. A organização do trabalho da enfermagem na perspectiva dos trabalhadores de um hospital escola. Revista Texto \& Contexto Enfermagem. 2002; 11 (1): 187-205.

5. Svaldi J, Dei S, Filho L, Lunardi WD. Métodos, ferramentas e técnicas da gestão da qualidade de total: aplicação na organização do trabalho da enfermagem em um serviço de pronto atendimento da enfermagem. Revista Texto \& Contexto Enfermagem. 2003; 12 (4): 510-18.

6. Passos JP, Ciosak SI. A concepção dos enfermeiros no processo gerencial em unidade básica de saúde. Revista da Escola de Enfermagem Universidade de São Paulo. 2006; 40(4):464-68.

7. Machado MED. Amamentação: a ação da equipe de enfermagem na perspectiva da fenomenologia social de Alfred Schütz [dissertação de mestrado]. Rio de Janeiro: Universidade do Estado do Rio de Janeiro; 2005.

8. Silva ALAC, Rodrigues BMRD. A sociologia fenomelógica de Alfred Schütz e a possibilidade de compreender em enfermagem. Revista de Enfermagem da Universidade do Estado do Rio de Janeiro. 1997; 5:475-78. 
9. Oliveira TJ. O cuidar de enfermagem à criança vítima de violência intrafamiliar: uma análise fenomenológica [dissertação de mestrado]. Rio de Janeiro: Universidade do Estado do Rio de Janeiro. 2004.

10. Waldow VR. Cuidado humano: o resgate necessário. Porto Alegre (RS): Sagra Luzzatto; 1998.

11. Silva SH, Fernandes RAQ, Gonçalves VLM. A enfermagem e a administração de recursos materiais: experiências do Hospital Universitário da USP. Hosp Adm Saúde. 1990; 14 (1): 34-8.

12. Geovanini TAM, Dornelles S, William CAM. História da enfermagem: Rio de Janeiro: Livraria e Editora Revinter; 1995.

13. Potter PA, Perry AG. Grande tratado de enfermagem prática: clínica e prática hospitalar. São Paulo: Editora Santos; 1998.

14. Barbier RA. A pesquisa-ação. Brasília (DF): Editora Plano; 2002, p.

15. Santos I, Gauthier J, Figueiredo NMA, Petit SH. Prática da pesquisa em ciências humanas e sociais: abordagem sociopoética. Rio de Janeiro: Editora Atheneu; 2005.
16. Sobral VT, Santos I, Araújo MFS. Sensibilizando la formación del cuidado. Enfermería Global (España). 2003; 3 (1): 20-5.

17. Watson J. Enfermagem pós-moderna e futura: um novo paradigma da enfermagem. Loures (Po): Lousociência; 2002.

18. Santos I. Cuidando do educando: a sociopoética sensibilizando a formação do cuidador. R Enferm UERJ. 2007;15 (1): 148-13.

19. Kurcgant P. Administração em enfermagem. São Paulo: EPU; 1991, p. 237.

20. David HMSL, Matos HS, Silva TS, Dias MG. Tabagismo e saúde da mulher: uma discussão sobre as campanhas de controle do tabaco; R Enferm UERJ. 2006; 14:412-17.

21. Weber M. Textos selecionados. Tradução de Maurício Tragtenberg. $2^{a}$ ed. São Paulo: Editora Abril Cultural; 1980, p. 238.

22. Husserl E. Husserl os pensadores história das grandes idéias do mundo ocidental: investigações lógicas. São Paulo: Editora Abril Cultural; [s.d.], p. 404.

23. Ministério da Saúde (Br). Programa Nacional de Humanização da Assistência Hospitalar. Brasília (Br): Ministério da Saúde; 2004, p. 23. 